\title{
A rare case of acute promyelocytic leukemia with ider(17)(q10)t(15;17)(q22;q21) and favorable outcome
}

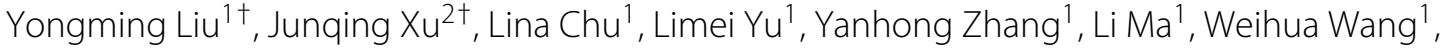 \\ Yangyang Zhang ${ }^{1}$, Yimin $\mathrm{Xu}^{1}$ and Riming Liu ${ }^{1 *}$
}

\begin{abstract}
Background: Chromosomal rearrangements in addition to $\mathrm{t}(15 ; 17)$ have been reported in $25-40 \%$ of APL patients, with a large predominance of trisomy 8 . Other abnormalities are far less frequent, particularly as ider(17), and the prognostic significance is still unclear.

Case presentation: We present the case of a patient with $t(15 ; 17)(q 22 ; q 21)$, der(15)t(15;17) and ider(17)(q10)t(15;17)(q22;q21). In particular, the RT-PCR result for PML-RARA of this patient was a false negative and mutational analysis of AML-related genes showed SNP rs2454206 in the TET2 gene and yielded negative findings in other genes including AML1, ASXL1, CEBPA, DNMT3A, FLT3, KIT, NPM1, TP53, and U2AF1. After the early usage of arsenic trioxide combinated with ATRA and vigorous supportive treatment to maintain PLT $\geq 30 \times 10^{9} / \mathrm{L}$ and FIB $>1500$ $\mathrm{mg} / \mathrm{L}$, this patient was under MMR and HCR without any clinical symptoms or signs until now.

Conclusion: False negative reslults of RT-PCR analysis for PML-RARA are rare in APL and ider(17) is even more infrequent. To our knowledge, this is the first reported case of APL with ider(17) and false negative RT-PCR analysis results. The role of ider(17) in APL is still an ongoing investigation and limited by the small number of published cases. The patient reported here benefited from vigorous supportive treatment during the combination of ATRA and arsenic trioxide in induction chemotherapy and the clinical outcome was favorable.
\end{abstract}

Keywords: FISH, RT-PCR, APL, PML-RARA, Ider(17)(q10)t(15;17)(q22;q21)

\section{Background}

Acute promyelocytic leukemia (APL) is a subtype of acute myeloid leukemia (AML) with specific clinical and biological features. APL is identified by distinctive morphology and characterized by the specific chromosomal rearrangement $\mathrm{t}(15 ; 17)$ (q22; q21), resulting in the fusion of the retinoic acid alpha gene (RARA) on 17q21 to the promyelocytic leukemia gene (PML) on 15q22 [1]. The disease used to be one of the most fatal forms of acute leukemia

\footnotetext{
*Correspondence: 719112295@qq.com

${ }^{\dagger}$ Yongming Liu and Junqing Xu contributed equally to this work.

${ }^{1}$ Clinical Laboratory, Qindao University Medical College Affiliated Yantai

Yuhuangding Hospital, No. 20, Yuhuangding East Road, 264000 Yan Tai, China

Full list of author information is available at the end of the article
}

with poor outcomes. The introduction of all-trans retinoic acid (ATRA) in combination with arsenic trioxide and other chemotherapies has resulted in complete remission rates $>90 \%$ and long-term remission rates above $80 \%[2]$. However, there is still a small population of APL patients who have a poor prognosis [3-5]. To the best of our knowledge, specific cytogenetic abnormalities can cause a change in treatment response, relapse and clinicopathological characteristics [6]. Chromosomal rearrangements in addition to $\mathrm{t}(15 ; 17)$ have been reported in $25-40 \%$ of APL patients, with a large predominance of trisomy 8. Other abnormalities are far less frequent, particularly ider(17). The prognostic significance of these abnormalities is still unclear [3, 7]. Here, we present the case 
of a patient with $\mathrm{t}(15 ; 17)(\mathrm{q} 22 ; \mathrm{q} 21), \operatorname{der}(15) \mathrm{t}(15 ; 17)$ and $\operatorname{ider}(17)(\mathrm{q} 10) \mathrm{t}(15 ; 17)(\mathrm{q} 22 ; \mathrm{q} 21)$ identified by conventional cytogenetics study and dual-color dual-fusion fluorescence in situ hybridization, and dual-color break apart fluorescence in situ hybridization methods.

\section{Case presentation}

In December 2018, a 26-year-old man was brought to Yantai Yuhuangding Hospital with the chief complaint of periumbilical pain and bleeding gums for 7 days. He had a fever of 38.7 Celsius. The initial complete blood count showed a hemoglobin level of 65 $\mathrm{g} / \mathrm{L}$, platelet count of $12 \times 10^{9} / \mathrm{L}$, and white blood cell count of $4.03 \times 10^{9} 9 / \mathrm{L}$ with $59 \%$ promyelocytes, $6 \%$ neutrophils, 34\% lymphocytes, and 1\% neutrophilic metamyelocytes. Coagulation tests revealed that the prothrombin time, thrombin time and activated partial thromboplastin time were within the normal ranges, but the fibrinogen and D-dimer levels increased to $4.52 \mathrm{~g} / \mathrm{L}$ and $20.77 \mathrm{mg} / \mathrm{L}$, respectively. The bone marrow aspirate showed hypercellular marrow with $83 \%$ promyelocytes that had numerous granules, increased Auer rods, and strong myeloperoxidase activity (Fig. 1). Flow cytometry analysis of the bone marrow showed that $81.1 \%$ of blasts were strongly positive for CD38dim, CD13, myeloperoxidase, CD123, CD33str, and CD117, with partial expression of CD9, CD19, CD64, cCD79a and CD11b, whereas CD34, CD7, HLA-DR, CD36, CD56, CD4, CD14, CD15, CD16, CD71, CD2, CD5, CD105, CD20, mCD3, CD8, CD10, and $\mathrm{cCD} 3$ were negative. Mutational analysis of AML related genes using a highthroughput DNA sequencing technique showed the single nucleotide polymorphism (SNP) rs2454206 in the TET2 gene (TET2:NM_001127208:exon11:c.A5284G:p.I1762V

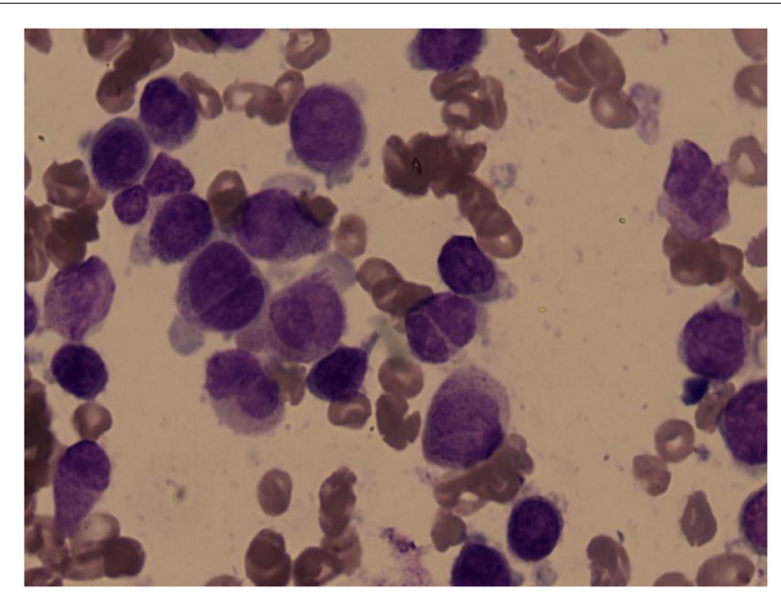

Fig. 1 Bone marrow morphology at initial diagnosis. The bone marrow aspirate showed hypercellular marrow with increased abnormal promyelocytes, which had numerous granules, increased Auer rods, and visible of round or oval, distorted, folded nucleus rs2454206, 48.28\%) and yielded negative findings in other genes including $A M L 1, A S X L 1, C E B P A$, DNMT3A, FLT3, KIT, NPM1, TP53, and U2AF1. Classical cytogenetic analysis showed 17 metaphase cells with 46,XY,der(15)t(15;17)(q22;q21),ider(17)(q10)t(15;17) (q22;q21); 1 metaphase cell with 46,XY,t(15;17)(q22;q21); and 2 normal cells according to ISCN2016 [8] (Fig. 2a and b). Fluorescence in situ hybridization (FISH) using a Vysis LSI PML/RARA dual color, dual fusion translocation probe (Abbott Molecular, Des Plaines, IL) indicated nuc ish $(P M L \times 4),(R A R A \times 4),(P M L$ con $R A R A \times 3)[400 / 500],(P M L \times 3),(R A R A \times 3)$, and $(P M L$ con $R A R A \times 2)[25 / 500$ ] (Fig. 2c). FISH using a Vysis LSI RARA dual color break apart rearrangement probe revealed nuc ish $\left(5^{\prime} R A R A \times 4\right),\left(3^{\prime} R A R A \times 2\right),\left(5^{\prime} R A R A\right.$ con5'RARA $\times 2)$ [400/500], (5'RARA $\times 2), \quad\left(3^{\prime} R A R A \times 2\right)$, (5'RARA con5'RARA $\times 1$ )[25/500] (Fig. 2d), which is consistent with the result of FISH using the PML/RARA dual color, dual fusion translocation probe. Reverse transcriptase-polymerase chain reaction (RT-PCR) analysis for the $P M L-R A R A$ rearrangement unexpectedly showed a false negative result; therefore, sequence analysis of PML-RARA were conducted. Sequencing of this sample confirmed that there was a fusion between $P M L$ exon 6 and RARA intron 3 (variant form)(Fig. 3). According to the sequence analysis results, new probes were designed, and the RT-PCR analysis for the PML-RARA rearrangement showed a positive result. This patient was diagnosed with APL according to bone marrow morphology, immunophenotyping, and molecular biology studies. He was treated with ATRA at a dose of $25 \mathrm{mg} / \mathrm{m} 2$ per day and arsenic trioxide $\left(\mathrm{As}_{2} \mathrm{O}_{3}\right)$ at a dose of $0.16 \mathrm{mg} / \mathrm{kg}$ per day for 30 days under the Chinese Guidelines for the diagnosis and treatment of acute promyelocytic leukemia (2018) [9].

After completing induction chemotherapy, the blood cell counts returned to normal levels and the bone marrow aspirate showed hematological complete remission (HCR) but not major molecular remission (MMR) with the detection of PML-RARA by FISH and RT-PCR with the newly designed probes. Then, one cycle of the DA regimen (daunorubicin $60 \mathrm{mg} / \mathrm{m} 2 / \mathrm{d}, \mathrm{d} 1-3$; cytarabine 200 $\mathrm{mg} / \mathrm{m} 2 / \mathrm{d}, \mathrm{d} 1-7)$ and another cycle of the DA regimen (daunorubicin $45 \mathrm{mg} / \mathrm{m} 2 / \mathrm{d}$, d1-3; cytarabine $1 \mathrm{~g} / \mathrm{m} 2 / 12 \mathrm{~h}$, d1-4) were administrated to the patient as consolidation chemotherapy. The patient is currently under MMR and HCR without any clinical symptoms or signs until now.

\section{Discussion and conclusion}

APL is a unique subtype of acute myeloid leukemia and is characterized by a balanced reciprocal translocation between chromosomes 15 and 17. To the best of our knowledge, the ider(17)(q10)t(15;17)(q22;q12) has been found in approximately $1 \%$ of the reported secondary 


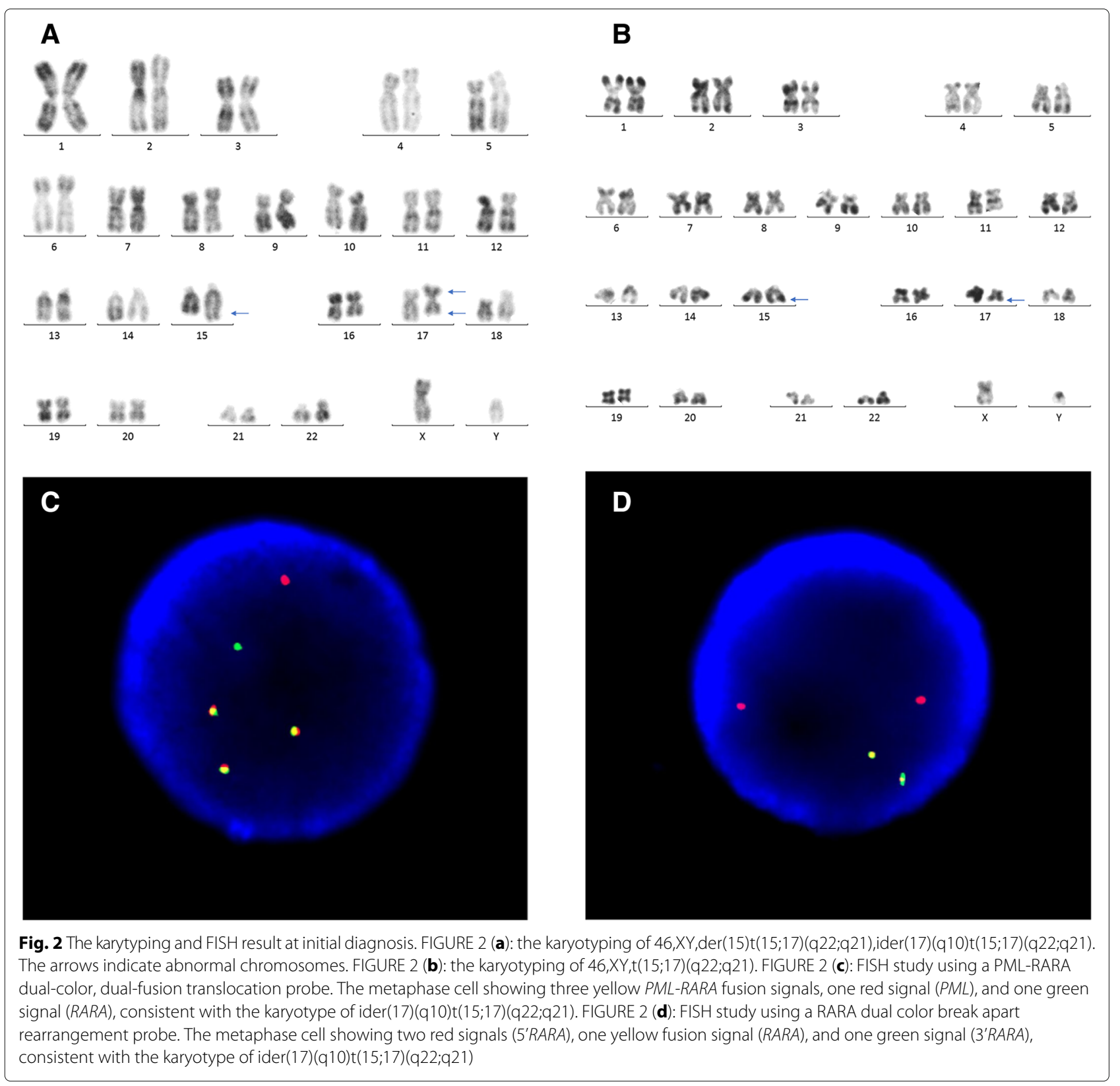

cytogenetic abnormalities in APL patients, which has been reported only in 85 APL cases worldwide [1, 3]. Here, we described a rare APL case with a false negative RT-PCR result and a TET2 SNP rs2454206 that had ider(17)(q10)t(15;17)(q22;q12).

SNP rs2454206 is common in the TET2 gene, which plays a role in epigenetic regulation of myelopoiesis. Wang et al reported that TET2 SNP rs2454206AG/GG is associated with improved overall survival and event-free survival in AML patients with intermediate-risk cytogenetics features [10, 11]. To the best of our knowledge, this mutation has not been reported in APL with ider (17)(q10)t $(15 ; 17)(\mathrm{q} 22 ; \mathrm{q} 12)$ and its prognostic significance in APL remains unclear.

RT-PCR is considered the most sensitive tool for diagnosing $P M L-R A R A$-positive leukemia. However, the false negative rate of RT-PCR for diagnosing $P M L-R A R A$ is approximately 1\% [9]. To the best of our knowledge, this is the first case report of a false negative for diagnosing $P M L-R A R A$ in a patient that had $\operatorname{ider}(17)(q 10) t(15 ; 17)(q 22 ; q 12)$. In our experience, it is not advisable to rely only on RT-PCR for the diagnosis of PML-RARA-positive APL and FISH analysis was equivalent to or even better than RT-PCR. Although FISH is a 


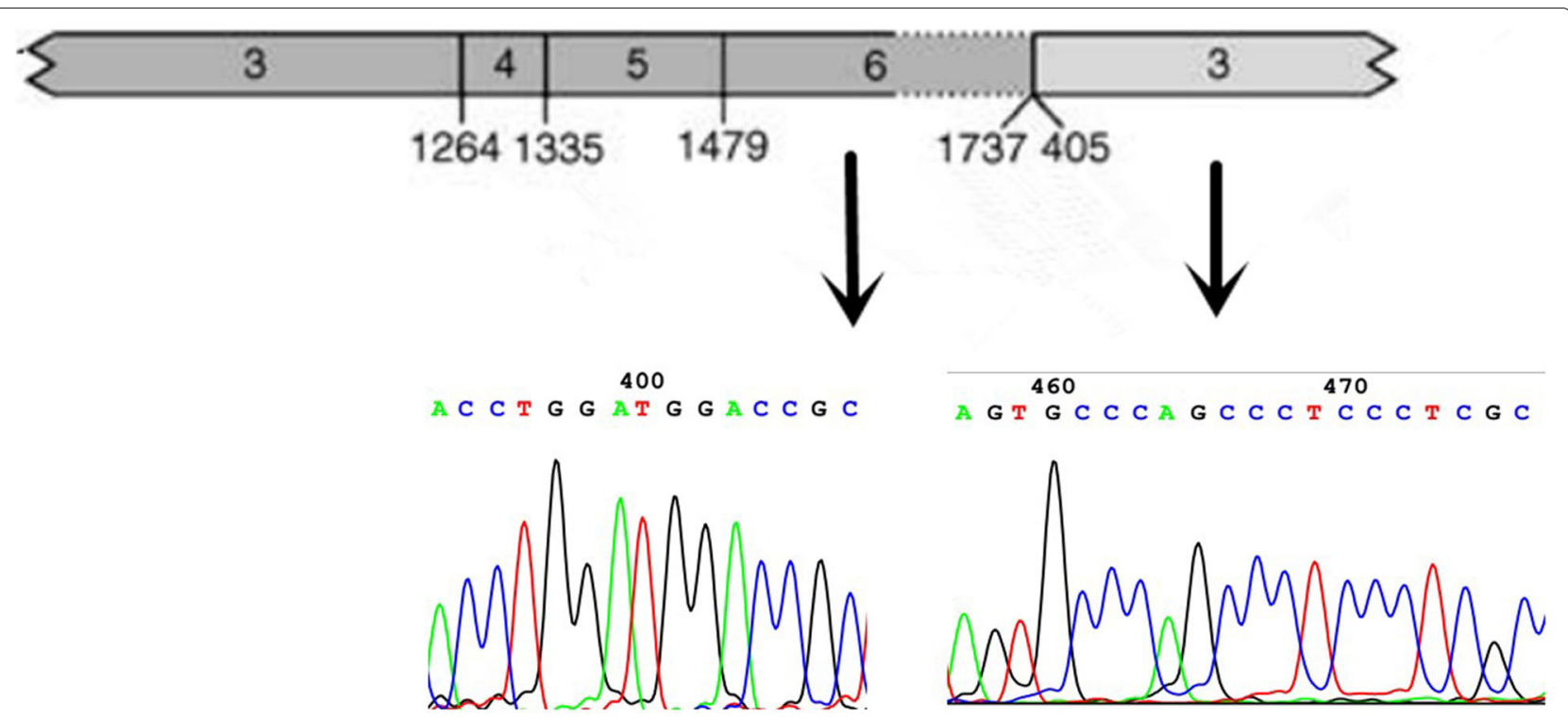

Fig. 3 Sequencing analysis of PML-RARA fusion transcripts at initial diagnosis. Diagrammatic representation and sequencing information of PML-RARA fusion transcripts of the patient. PML transcripts consisting of exon 6 joined to RARA intron 3 (variant form)

highly specific confirmatory test for the diagnosis of APL, it cannot easily analyze low-level signal patterns, such as $2-5 \%$, to determine the clinical significance of the results when used for the detection of minimal residual disease [12]. Therefore, RT-PCR and FISH are recommended to be performed as parallel tests for the diagnosis of APL.

The frequency of the clone with ider(17)(q10)t(15;17)(q22;q12) was higher than that of the clone with $t(15 ; 17)$ alone, which is consistent with the findings of other studies. Manola et al suggest that the duplication of $\operatorname{der}(17 \mathrm{q})$, overrepresentation of the $R A R A-P M L$ fusion gene and loss of the normal tumor suppressor gene $P 53$ mapped on $17 \mathrm{p} 13$ due to the loss of $17 \mathrm{p}$, which plays an important role in oncogenesis and serves as a poor prognostic factor in leukemia, may contribute to this growth advantage [13].

Some reports suggested that $\operatorname{ider}(17)(\mathrm{q} 10) \mathrm{t}(15 ; 17)$ (q22;q12) might be a poor prognostic factor in APL $[1,3,12-15]$. Therefore, some reports showed that the clinical course of patients with ider(17)(q10)t(15;17) (q22;q12) did not seem to differ from that of the typical $t(15 ; 17)(q 22 ; q 12) \quad[13,15]$. The role of $\operatorname{ider}(17)(q 10) t(15 ; 17)(q 22 ; q 12)$ in APL is still an ongoing investigation and is limited by the small number of published cases. In our experience, the clinical outcomes of this patient may be due to the following 3 points. First, this patient was classified in the low-risk group at the initial diagnosis stage according to the 2017 NCCN guideline for AML, which correlates with the therapeutic effects, clinical outcomes and relapse for APL. Second, the early usage of arsenic trioxide combined with ATRA, has emerged as the new standard of care for patients with low-to-intermediate risk APL [16]. Last, continuous transfusion of platelet concentrates and fresh frozen plasma was conducted to maintain PLT $\geq 30 \times 10^{9} / \mathrm{L}$ and FIB $>1500 \mathrm{mg} / \mathrm{L}$. APL is now considered to be the most curable subtype of acute myeloid leukemia in adults. Nevertheless, APL remains associated with a significant incidence of early death related to the characteristic bleeding diathesis [16]. Therefore, we infer that the patient reported here may benefit from vigorous supportive treatment during the combination of ATRA and arsenic trioxide in induction chemotherapy. However, more case reports and systematic analyses are needed to help us better understand the clinical, cytogenetic and molecular features and prognostic significance of $\operatorname{ider}(17)(q 10) t(15 ; 17)(q 22 ; q 12)$ in APL patients.

\section{Abbreviations \\ PML: promyelocytic leukemia gene; RARA: retinoic acid alpha gene; AML: acute myelocytic leukemia; APL: acute promyelocytic leukemia; FISH: fluorescence in situ hybridization; HCR: hematological complete remission; MMR: major molecular remission RT-PCR: reverse transcriptase-polymerase chain reaction; SNP: single nucleotide polymorphism;}

\section{Acknowledgements}

I would like to express my gratitude to all those who have helped me during the writing of this thesis.

\section{Authors' contributions}

Conceived and designed the study: Riming Liu. Performed the study: Yongming Liu. Analyzed the data: Junging Xu. Contributed reagents/materials/analysis tools: Lina Chu, Limei Yu, Yanhong Zhang, Li Ma, Hui Xiao, Weihua Wang, Yangyang Zhang, Yimin Xu

\section{Funding}

None. 


\section{Availability of data and materials}

The data sets used and/or analyzed during the current study are available from the corresponding author on reasonable request. All authors read and approved the final manuscript.

\section{Ethics approval and consent to participate}

All procedures performed in this study were in accordance with the ethical standard of the institutional and national research committee and with the 1964 Helsinki declaration and its later amendments or comparable ethical standards

\section{Consent for publication}

Informed written consent for publication was obtained from the patient. A copy of the consent form is available.

\section{Competing interests}

The authors declare that they have no competing interests.

\section{Author details}

${ }^{1}$ Clinical Laboratory, Qindao University Medical College Affiliated Yantai Yuhuangding Hospital, No. 20, Yuhuangding East Road, 264000 Yan Tai, China. ${ }^{2}$ Department of Hematological, Qindao University Medical College Affiliated Yantai Yuhuangding Hospital, No. 20, Yuhuangding East Road, 264000 Yan Tai, China.

Received: 7 January 2020 Accepted: 9 March 2020

Published online: 10 April 2020

\section{References}

1. Hu X, Ai G, Meng X, et al. An ider(17)(q10)t(15;17) with spliced long-type pml-rara fusion transcripts in a case of acute promyelocytic leukemia. Cancer Genet. 2014;207:253-7.

2. Testa U, Lo-Coco F. Prognostic factors in acute promyelocytic leukemia: strategies to define high-risk patients. Ann Hematol. 2016;95:673-80.

3. Kim M, Cho S, Lim G, et al. A rare case of microgranular acute promyelocytic leukemia associated with ider(17)(q10)t(15;17) in an old-age patient. Korean J Lab Med. 2011;31:86-90.

4. Lengfelder E, Saussele S, Weisser A, et al. Treatment concepts of acute promyelocytic leukemia. Crit Rev Oncol Hematol. 2005;56:261-74.

5. Sirulnik L, Stone R. Acute promyelocytic leukemia: current strategies for the treatment of newly diagnosed disease. Clin Adv Hematol Oncol. 2005;3:391-397429.

6. Cervera J, Montesinos P, Hernández-Rivas J, et al. Additional chromosome abnormalities in patients with acute promyelocytic leukemia treated with all-trans retinoic acid and chemotherapy. Haematologica. 2010;95:424-31.

7. Kim M, Lee $\mathrm{S}$, Park H, et al. Two distinct clonal populations in acute promyelocytic leukemia, one involving chromosome 17 and the other involving an isochromosome 17. Cancer Genet Cytogenet. 2010;197: 185-8.

8. Jean M-J, Simons A, Schmid M. An International System for Human Cytogenetic Nomenclature 2016. Basel: Karger Medical and Scientific Publishers; 2016.

9. Chinese Society of Hematology and Chinese Medical Doctor Association, Chinese Medical Association, Chinese Medical Doctor Association. Chinese guidelines for diagnosis and treatment of acute promyelocytic leukemia 2018. Zhonghua Xue Ye Xue Za Zhi. 2018;39:179-83.

10. Wang $X$, Chen $X$, Yang Z, et al. Correlation of tet2 snp rs 2454206 with improved survival in children with acute myeloid leukemia featuring intermediate-risk cytogenetics. Genes Chromosom Cancer. 2018;57: 379-86.

11. Kutny M, Alonzo T, Gamazon E, et al. Ethnic variation of tet2 snp rs2454206 and association with clinical outcome in childhood aml: a report from the children's oncology group. Leukemia. 2015;29:2424-6.

12. Kim M, Yoon H, Cho S, et al. ider(17)(q10)t(15;17) associated with relapse and poor prognosis in a pediatric patient with acute promyelocytic leukemia. Cancer Genet Cytogenet. 2010;201:116-21.

13. Manola K, Karakosta M, Sambani C, et al. Isochromosome $\operatorname{der}(17)(q 10) t(15 ; 17)$ in acute promyelocytic leukemia resulting in an additional copy of the rara-pml fusion gene: report of 4 cases and review of the literature. Acta Haematol. 2010;123:162-70.
14. Xu L, Zhao W, Xiong S, et al. Molecular cytogenetic characterization and clinical relevance of additional, complex and/or variant chromosome abnormalities in acute promyelocytic leukemia. Leukemia. 2001;15: 1359-68.

15. He $Y$, Wang $P$, Liang $K$, et al. A pediatric acute promyelocytic leukemia with a rare karyotype of ider(17)(q10)t(15;17) and favorable outcome: A case report. Med (Baltim). 2015;94:1778.

16. Coombs C, Tavakkoli M, Tallman M. Acute promyelocytic leukemia: where did we start, where are we now, and the future. Blood Cancer J. 2015;17:304.

\section{Publisher's Note}

Springer Nature remains neutral with regard to jurisdictional claims in published maps and institutional affiliations.
Ready to submit your research? Choose BMC and benefit from:

- fast, convenient online submission

- thorough peer review by experienced researchers in your field

- rapid publication on acceptance

- support for research data, including large and complex data types

- gold Open Access which fosters wider collaboration and increased citations

- maximum visibility for your research: over $100 \mathrm{M}$ website views per year

At BMC, research is always in progress.

Learn more biomedcentral.com/submissions 